\title{
Effect of Osteoblasts Cell Adhesion Behavior on Biomaterial Surfaces by Atomic Force Microscope
}

\author{
Afrina Khan Piya ${ }^{1}$, Munshi Muhammad Raihan ${ }^{1}$, Md Alamgir Hossain ${ }^{2,}$ * \\ ${ }^{1}$ Department of Mechanical Engineering, Nagaoka University of Technology, Nagaoka City, Japan \\ ${ }^{2}$ Department of Mechanical Engineering, Military Institute of Science and Technology, Dhaka, Bangladesh
}

Email address:

afrina.mist@gmail.com (A. K. Piya), raihan.mist@gmail.com (M. M. Raihan), ahossain@me.mist.ac.bd(Md A. Hossain)

${ }^{*}$ Corresponding author

\section{To cite this article:}

Afrina Khan Piya, Munshi Muhammad Raihan, Md Alamgir Hossain. Effect of Osteoblasts Cell Adhesion Behavior on Biomaterial Surfaces by Atomic Force Microscope. Advances in Applied Sciences. Vol. 5, No. 1, 2020, pp. 1-10. doi: 10.11648/j.aas.20200501.11

Received: October 26, 2019; Accepted: November 29, 2019; Published: March 23, 2020

\begin{abstract}
Atomic force microscopy (AFM) is a great scientific invention that can visualize cell morphology in aqueous environment and provide information to investigate cell biomechanics at a high spatial resolution in a controlled environment with force sensitivity. Contemporary AFM techniques permit solving a number of problems of cell biomechanics due to synchronized evaluation of the local mechanical properties. For characterizing mechanical properties force spectroscopy is used that provides information on cellular structures including cytoskeleton structure and morphology. For the success of biomedical implant, the most crucial factor is biocompatibility and osteo-conductivity of the implant material that can be characterized by the change in mechanical properties of cellular filaments and nucleus determined by exploiting atomic force microscope techniques. Hydroxyapatite is a bioactive material in bio-ceramics, hence used for fillers, bone grafts and metallic implant coating. Recently developed HAp/amino acid fluorescent complexes could be a significant candidate to be used for dental implant, had shown antibacterial properties with visible light irradiation. This study aims at revealing murine osteoblasts cell (MC3T3) adhesion behavior on the HAp coating and HAp/amino acid complexes. The AFM revealed that no significant changes were observed in mechanical properties of the osteoblasts cells when adhered on electrochemically deposited HAp coating and HAp/amino acid ligands complex coating. SEM and EDX analysis revealed cell morphology were identical for HAp and HAp/amino acid ligands complex coating. Such characteristics are desirable for the success of implant biomaterial coating that can preserve both antibacterial property and cell adhesion behavior.
\end{abstract}

Keywords: Dental Implant, Antibacterial Property, HAp/Amino Acid Complex, Atomic Force Microscopy (AFM), Cell Elasticity, CIP, Cytoskeleton Dynamics, Biomaterials

\section{Introduction}

Titanium and titanium-based alloys have been widely used for load bearing applications in biomedical devices, such as surgical or dental implants [1-3]. Ti-6Al-4V ELI (Extra Low Interstitial) (ASTM F136) alloy, has been specifically applied for both surgical implants or aeronautics applications. Though the strength, fracture toughness and corrosion resistance of the alloy are sufficiently high as the composition of surgical implants, a stress shielding effect due to the large gaps in Young's modulus between the one of the Ti alloy with the other of surrounding cancellous bones, which may lead to reduction of bone density and its strength, has been frequently occurred. The stress shielding effect can promote micro motions between the surgical implants with surrounding bones during mechanical loading, and the damages can subsequently lead to osteolysis, bone fracture or loosening of the implants. In order to suppress such the side effects by incompatibility of artificial materials with the natural bone, biomaterials considering biocompatibility have been developed. In the case of $\mathrm{Ti}$ alloys, have been actively developed in order to obtain lower values of Young's modulus of the alloys, which can be closer to the one of natural bones [2-4]. Biological safety tests, which can evaluate cytotoxicity or safety in relatively short-term compared with the one in considering 
biocompatibility of biomedical devises, are only determined in ISO 10993 series. On the other hand, there has been no rationally regulated method to evaluate biocompatibility of biomaterial [5-11]. The requirement for biocompatibility of biomedical devises is recently skyrocketing due to extended service lives of the devises corresponding to longer life expectancy.

Surface properties of materials is one of the critical factors in considering biocompatibility of materials. For example, Ti alloy cannot directly bond with natural bone and therefore Hydroxyapatite (HAp) coating is deposited onto the surfaces [12-15]. HAp, which possesses similar chemical composition with the one of natural bone (a composite structure of calcium phosphate ceramic and collagen) can mediate direct bonds between natural bones with the Ti alloy by promoting precipitation of calcium phosphate and subsequent crystallization on its surface [16]. Such the feature of HAp coating is called osteoconductive [17], which can be regarded as a specific type of hard tissue adhesiveness. HAp coating can also change physical, mechanical and electrochemical property compared with those of Ti alloy. Such the features are known to influence cell adhesion behavior onto the surface [18-19], which is also an important aspect in considering biocompatibility because biological reactions such as bone remodeling or immunological reaction are regulated by specific types of cells, e.g., osteoclast/osteoblast cells and macrophages [20]. Multifunctional coating, which can possess both osteoconductivity and antibacterial property, has been recently investigated in order to increase coated components in surgical implants [20, 21]. Multifunctional coating can be developed on the basis of profound understanding on the regulated cell adhesion behavior; preferable cells for biological reactions should be bonded more strongly and undesirable bacteria/virus must be regulated.

Atomic force microscopy (AFM) is one of nanoscopic imaging method to observe surface features of materials by detecting atomic forces between the surface and a cantilever $[22,23]$. In order to reveal microscopic mechanism of biocompatibility, more detailed assessing method to evaluate the extents of adhesiveness of the cells onto the surfaces of biomaterials are required. Electrochemical deposition is an effective means of fabricating HAp coatings as a liquid based process on intricate geometry substrates [24]. The deformation of cantilever is measured and controlled by an optical lever using a semiconductor laser (typical wavelength is $633 \mathrm{~nm}$ ). It can observe surface morphology, electric property and magnetic property of materials [23-25]. Force curve measurement to evaluate mechanical properties is also AFM technology has recently been applied to assess cell adhesion behavior [24-29]. Zuttion et. al. used atomic force microscopy to study the effect of glycoclusters on the bacteria-cell interaction and observed a decreased detachment force of the bacteria (Pseudomonas aeruginosa (PA))-cell (globotriaosylceramide (Gb3)) interaction [30]. Zhang et. al. also applied AFM to measure interaction forces between $E$. coli bacterias and NPs immobilized on surfaces in an aqueous environment [31]. Huang et. al. demonstrated that adhesive force of $E$. coli bacteria in biofilms were approximately 3.0-0.4 N [32]. Those studies used immobilized bacteria onto the surfaces of cantilever and then viability of bacteria is challenged [30, 31]. Tanaka et. al. pointed out the importance of intermediate water in discussing biocompatibility of materials [33].

Force curve measurements to assess adhesion behavior of larger osteoblast cells have also been investigated [34-37], however, measured mechanical properties, especially adhesion forces were varied. Caneva Soumetz et. al. tried a nano scratching test using a hard cantilever for osteoblast cells and the detected adhesive forces were in the range of 30-90 pN [38, 39]. Researchers recently developed HAp complex coating, which can be assumed the great changes in mechanical properties of surface by organic ligands [1, 40-43]. Such the HAp complex coating could promote antibacterial properties of Titania coating under visible-light irradiation, though the details of enhancement mechanisms have not been sufficiently understood. At least, the force curve measurements in aqueous environment using living cells are necessary to discuss the adhesion behavior [44, 45]. One of the assumptions can be considered that the HAp complex coating could promote cell adhesion behavior. However, effects of such the changes in mechanical properties of surface on cell adhesion behavior of osteoblast cells in an aqueous environment was not sufficiently revealed yet. Several deposition methods of HAp coating have been proposed, such as plasma spraying technology, electrophoresis and sputtering methods, sol-gel, biomimetic, chemical vapor deposition, ion implantation [46-49] etc.

This study aims at evaluating the mechanical properties of osteoblasts cells with HAp/amino acid complex coating by Atomic Force Microscopy. The newly fabricated HAp complex coating could enhance the antibacterial property [50-52], but it is necessary to obtain the reliability of the complex coating for the biocompatible aspect. Determining the changes in mechanical properties i, e. Young's modulus value could provide information about the changes in the cellular body. AFM is an effective tool to certify the cellular dynamic information. HAp coating layer was fabricated by electrochemical deposition process on Ti-6Al-4V surface. HAp/amino acid complexes were then formed using Cold Isostatic Pressing Process. Fluorescence microscope observation was performed after $24 \mathrm{hrs}$. Of immersion testing at $37^{\circ} \mathrm{C}$ in distilled water in order to certify the formation of the complexes. AFM measurement was conducted to observe changes in surface morphology and mechanical properties of Osteoblasts cells adhered over the HAp and HAp complexes. Later on, SEM, EDS and Raman spectroscopy observation were being examined for determining the presence of Osteoblasts cells on the HAp and HAp complexes. Force curve measurement of osteoblast cells in culture medium was then conducted to discuss the mechanical properties of osteoblast cells on different types of HAp/amino acid ligands. 


\section{Experimental Procedure}

\subsection{Preparation of Titanium Alloy Substrate}

Ti-6Al-4V is used as the substrate material for this experiment. The plate of $10 \times 10 \times 3 \mathrm{~mm}^{3}$ was cut using a cutting machine. The plates were polished using emery papers from \#180, 250, 400, 800, 1000, 1500 and 2000 grit size respectively. The plates were finally buff-polished using diamond pastes $6,3,1 \mu \mathrm{m}$, respectively. After polishing the substrates were ultrasonically cleaned in ethanol by an ultrasonic cleaner (Model-ASU-6, China) for 10 minutes and dried in air. Surface profiles of polished specimens were observed by an optical microscope (Nikon ECLIPSE LV $150)$.

\subsection{Electrochemical Deposition of Hydroxyapatite Coating}

A typical three electrode electrochemical cell was used for the electrochemical deposition of hydroxyapatite coating. Ti-6Al-4V disc with an exposed area of approximately $1 \mathrm{~cm}^{2}$ was used as working electrode (WE), a saturated silver-silver chloride electrode $(\mathrm{Ag}-\mathrm{AgCl}$ in saturated $\mathrm{KCl}$ ) was used as reference electrode (RE) and a platinum foil with approximately $1 \mathrm{~cm}^{2}$ surface area was used as the counter electrode (CE). A Potentiostat (HZ-7000, Hokuto Denko Co. Ltd., Japan) was used for controlling the electrodeposition process operating in cyclic voltammetry mode from $0 \mathrm{~V}-1.4 \mathrm{~V}$ at the scanning rate of $0.2 \mathrm{mVs}$. The preparation for the electrolyte was done by dissolving $1.2 \mathrm{mM} \quad \mathrm{NH}_{4} \mathrm{H}_{2} \mathrm{PO}_{4}$ and $0.72 \mathrm{mM}$ $\mathrm{Ca}\left(\mathrm{NO}_{3}\right)_{2} \cdot 4 \mathrm{H}_{2} \mathrm{O}$ in ultra-pure distilled water with an initial $\mathrm{Ca}-\mathrm{P}$ ratio of 1.67. The $\mathrm{pH}$ of the solution was measured by a $\mathrm{pH}$ meter. The $\mathrm{pH}$ of the solution was adjusted to 6.0 to 6.5 by adding $0.01 \mathrm{M} \mathrm{NaOH}$, while the temperature was controlled at $75{ }^{\circ} \mathrm{C}$ using a magnetic hotplate stirrer [49]. After the electrochemical deposition process, the samples were rinsed and dried at room temperature in a vacuum drier.

\subsection{Fabrication of HAp/Amino Acids Complex Coating by Cold Isostatic Pressing (CIP)}

Phenylalanine (Phe) and Tryptophan (Tyr) (Kishida Chemical Co., Ltd. Osaka, Japan) are the candidate ligands of amino acids that were used to form the fluorescent complex of HAp/amino acid. Mass of $0.5 \mathrm{mg}$ of each types of powders of amino acid ligands were packed over the surface of HAp coated specimens. Then the specimen was wrapped tightly in a vacuum condition with a plastic wrap. To reduce the space between particle of HAp and amino acid, the plastic bags were then vacuum sealed by a vacuum machine for 90 seconds to remove remaining air from the plastic bag. The packed specimens were pressurized at $700 \mathrm{MPa}$ for holding times of $20 \mathrm{~min}$ each by using a CIP machine (Dr. CHEF, Kobe steel co. ltd., Japan).

\subsection{Fluorescent Microscope Observation}

Before measuring the fluorescence properties, the CIPed specimens were immersed in distilled water at $40{ }^{\circ} \mathrm{C}$ for 24 hours to remove the unreacted amino acid powder. Fluorescence was observed by a fluorescence microscope (BZ-8100, Keyence Co., ltd., Japan). The excitation conditions were as follows; wave lengths $360 \pm 20 \mathrm{~nm}$ (exposure time $0.5 \mathrm{~s}$ ), $470 \pm 20 \mathrm{~nm}$ (exposure time $0.5 \mathrm{~s}$ ) and $540 \pm 12.5 \mathrm{~nm}$ (exposure time $0.5 \mathrm{~s}$ ). The detection filters used were $460 \pm 20 \mathrm{~nm}, 535 \pm 25 \mathrm{~nm}$ and $605 \pm 27.5 \mathrm{~nm}$ respectively $[1,43]$.

\subsection{Surface Roughness Observation by AFM}

Surface roughness was observed by the Atomic Force Microscope (SPM-9700, Shimadzu co. ltd., Japan). Microcantilever (OMCL-TR800PSA-1, Olympus, co. ltd., Japan) in dynamic mode was used. The operating point was $0.165 \mathrm{~V}$, frequency was adjusted approximately $72-73 \mathrm{kHz}$ and the observation area was set as $1 \times 1 \mu \mathrm{m}$. The polished Ti-6Al-4V, coated HAp surface and fluorescent complex of $\mathrm{HAp} /$ amino acids were measured to evaluate the difference in the steps of formation of coating and complex.

\subsection{Osteoblasts Cell Culture}

Murine osteoblasts like cells MC3T-E1 (Riken Bio-resource Center, Japan) were cultured over the HAp and HAp/amino acid ligands complexes. The HAp coating specimens were sterilized in an autoclave at $120^{\circ} \mathrm{C}$ for 20 minutes and placed in culture medium in 24 well plates. The complex HAp/amino acids coating specimens were exposed to the UV light irradiation in clean bench for 2 hours before cell passage on the surface as the sterilization process. A clean bench (SANYO, MCV-711ATS) was used to place the specimens inside the well plate. The specimens were kept with the culture medium that contains 10\% FBS with D-MEM $(2 \mathrm{~mL})$ for 24 hours in incubator with $5 \%$ atmospheric $\mathrm{CO}_{2}$ and $100 \%$ relative humidity. The medium was then removed carefully and replaced with $2 \mathrm{~mL}$ of a fresh medium. The culture medium that contains the osteoblasts suspension was collected and seeded into cell culture plates. The specimens with cells were then cultured in an incubator with $5 \%$ of $\mathrm{CO}_{2}$ and $100 \%$ relative humidity for 24 hours.

\subsection{AFM Measurement in Living Osteoblasts Cell}

AFM technique is a quantitative study of mechanical characteristics of cells and tissues which is known as force-curve analysis. Force value and vertical deflection of the cantilever are being recorded, the probe approaches the surface under the observation zone at the fixed point and generally performs force-curve analysis. Basic principle of force curve measurement can be explained in few steps. The steps for the force curve measurement are as follows.

A cantilever is approaching onto the surface, 1) The cantilever probe on the cantilever tip is completely separated from the sample surface. 2) Slight attractive force is working on the tip of the cantilever and slight bending occurs. This is referred to as Jump in during force curve measurement. As 
the approaching is continued, the cantilever is subjected to a repulsive force and it is bent upwards. 4) In this position, the cantilever and the sample have the strongest contact and the repulsive force is at a maximum level.

Cantilever Releasing from the sample surface: 5) The acting force on the cantilever changes from repulsive to attractive. 6) In this position, an adhesive force is present between the cantilever with the sample and this attractive force is at a maximum. After this position, immediately the probe separates from the sample and this is referred to as the jump-out point. And then it comes to step 1 again. This illustration can be viewed in typical force curve diagram Figure 1 .

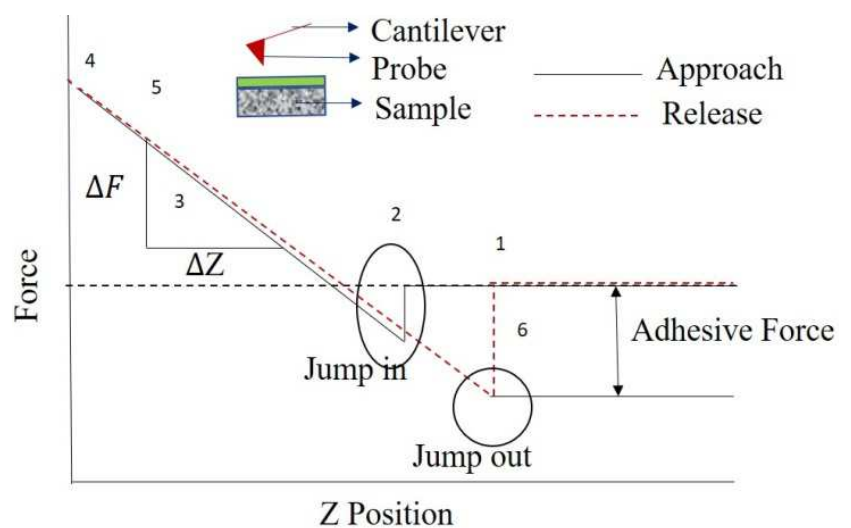

Figure 1. Schematic force curve diagram (According to manufacturer).

The AFM can be applied as micro indenter that probes the mechanical properties of the cell. The unadorned AFM tip was lowered onto the cell surface with the set rate, typically 5 $\mu \mathrm{m} / \mathrm{s}$. AFM tip exerted a force against the cell just after the contact that was proportional to the deflection of the cantilever. During the approach and withdrawal of the AFM tip, the deflection of the cantilever was recorded as a function of the piezoelectric translator position. To calibrate the deflection of the cantilever, force-indentation curves of the cells were derived from these records with the help of the surface of tissue culture dish. Assumptions that the cell is an isotropic elastic solid and the AFM tip is a rigid cone were made to estimate the Young's modulus value. According to this model, initially proposed by Hertz, the force $(F)$-indentation $(\alpha)$ relation is a function of Young's modulus of the cell $K$, and the angle formed by the indenter and the plane of the surface, $\theta$, as follows:

$$
F=\frac{K}{2\left(1-v^{2}\right)} \frac{4}{\pi \tan \theta} \alpha^{2}
$$

Young's modulus by least square analysis of the force-indentation curves were obtained by routines in the Igor Pro (Wave Metrics, Inc., Lake Oswego, OR) software package. It was assumed that the indenter angle, $\theta$, to be $55^{\circ}$ and Poisson ratio, $v$, to be 0.5 [50].

Force curve measurement process was performed in the contact mode in Nano 3D Mapping. During the force curve measurement, Micro Cantilever (OMCL-TR400PSA-1 Olympus co. Ltd, Japan) silicon nitride with
Gold/Chromium coating, whose resonance frequency was $24 \mathrm{kHz}$ with a force constant $0.15 \mathrm{~N} / \mathrm{m}$, was used. The length of the cantilever was 200 micrometer, cantilever width 27.9 micrometer, thickness $0.8 \mu \mathrm{m}$. The cantilever tip height is 2.9 micrometer, radius less than $20 \mathrm{~nm}$ and tip angle were less than 70 degrees. The tip angle between diagonal ridges was less than 90 degrees. The cantilever was swept in the vertical $(Z)$ direction close to the sample surface and the distances between the cantilever with the sample was adjusted. During this process the force acting on the cantilever is measured and hence the distance vs force graph is obtained by force curve measurement. This is known as the Force Curve.

In this work, the force curve measurement was carried out in liquid (Culture medium) conditions. The used samples for the force curve measurements were Osteoblasts cell adhered HAp coated specimen and HAp/amino acid complex specimens, respectively. Young's Modulus value, adhesive force was calculated from the force curve measurement. Here adhesive force is the force acting immediately prior to jump out position and hardness is measured by the slope $\Delta F / \Delta Z$. For Liquid condition distilled water was used. the cell testing the conditions were I-Gain: 200, P-Gain 0.00, Operating point $0.1 \mathrm{~V}$, scanning area $30 \times 30 \mu \mathrm{m}$, scanning rate $1 \mathrm{~Hz}$, liquid used DMEM. The temperature was kept at $120^{\circ} \mathrm{C}$ with room humidity $51 \%$. The scanner size was $125 \mu \mathrm{m}$. From the force curve measurement, mechanical properties could be measured between the osteoblasts cell adhered over the coated surface. Then the EDS analysis was done for the verification of the presence of cell.

\subsection{Structure of Cell Body by SEM}

After the atomic force microscope measurement, cultured cells were stained by $10 \%$ ionic liquid solution (HILEM $1 \mathrm{~L}$ 1000, Hitachi, Japan) to prevent the evaporation of the osteoblasts cells in vacuum chamber followed by air drying. The samples were observed using a Scanning Electron Microscope (JEOL JSM-5400LV, JEOL, Japan), followed by EDX analysis to confirm the cell morphology.

\subsection{Statistical Analysis}

ANOVA and multiple comparison by Holm's method were applied to the results of the cell viability test, the point indentations on individual MC3T3-E1 cells from each coating group was analyzed, and the mean apparent modulus was compared using $t$ test. The significance level was set to $p$ $<0.05$. All statistical analyses were performed in the R3.4.2 software.

\section{Results}

\subsection{Surface Morphology of HAp Coating Deposited by Electrochemical Method}

The cathodic reaction was observed in the electrochemical deposition hysteresis loop as shown in Figure 2.

Figure 3 shows the 3D optical microscope observation of 
the Ti-6Al-4V substrate after the electrochemical deposition process. Homogeneous layer of HAp coating was observed on the surface of Ti-6Al-4V substrate. Though, the HAp coating did not thoroughly deposit on the Ti-6Al-4V surface probably due to inhomogeneous reactions, the coverage ratio of HAp coating was sufficiently high.

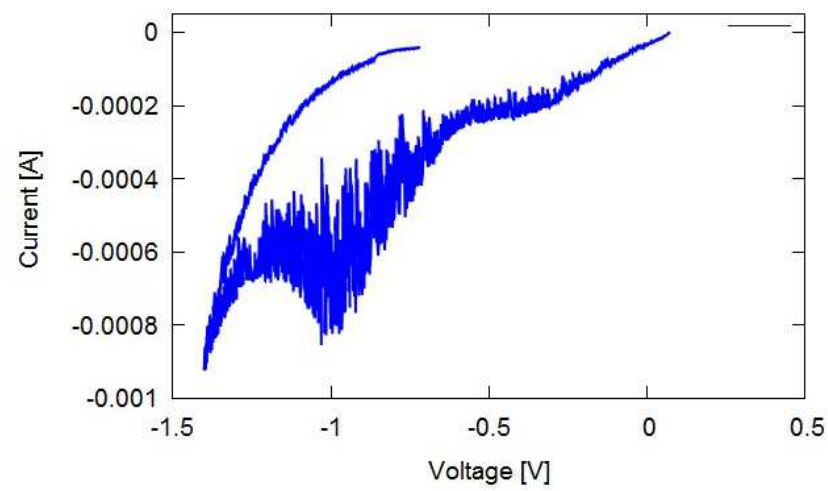

Figure 2. Cyclic Volumetric hysteresis of cathodic reaction of $\mathrm{CaPO}_{4}$ deposited on Ti-6Al-4V substrate.

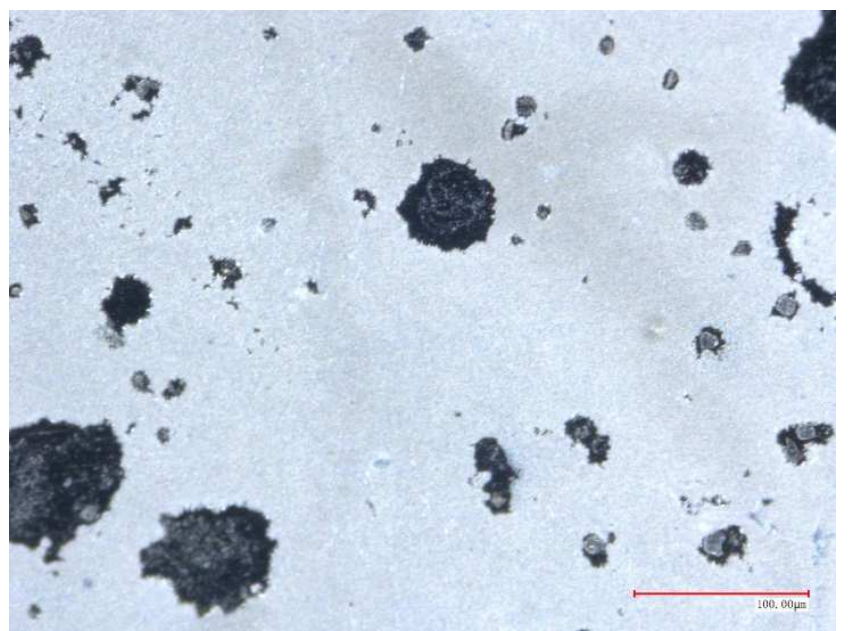

Figure 3. 3D optical microscope observation of the Ti-6Al-4V substrate after the electrochemical deposition process.

Surface sensitive technologies like AFM, requires surface roughness to in range on nanometer scale. Previous studies of AFM measurement for osteoblast cells generally used glass substrate as a model substrate. However, mechanical property of glass substrate and HAp coated Ti-6Al-4V substrate was quite different though the changes in mechanical properties greatly affected adhesion behavior [26]. This deposition method could provide suitably smooth HAp coating for AFM measurement as shown in Figure 3.

\subsection{Fluorescent Observation of HAp/Amino Acids Complex Coating}

The fluorescence microscope image of raw tryptophan and phenylalanine powders showed no fluorescence. The fluorescent microscope observation showed only the reflected light from the surfaces. The pure amino acid ligands tryptophan and phenylalanine does not possess any fluorescence property in visible-light region that was clearly observed in Figure 4.

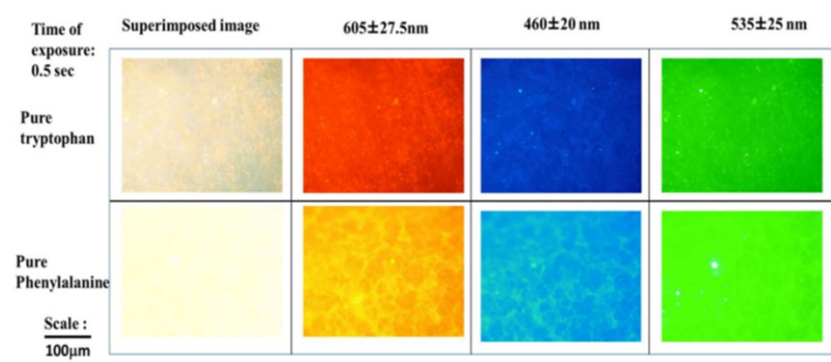

Figure 4. Fluorescence property observation for the pure tryptophan and pure phenylalanine powder.

Figure 5 shows the fluorescence microscope image of CIPed HAp coating, which demonstrated that HAp-amino acid complexes was successfully formed on the surface of HAp coating deposited by electrochemical process. HAp/tryptophan complex exhibited yellow fluorescence and HAp/phenylalanine complex consistently showed yellow fluorescence with red-shift one, which was commonly observed previously.

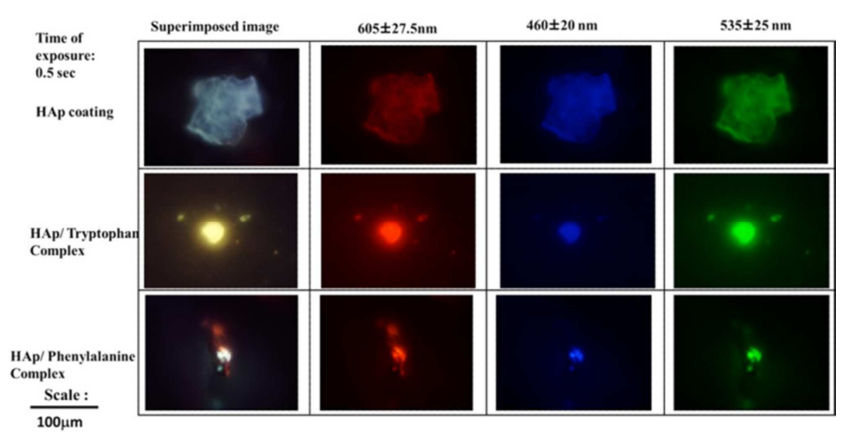

Figure 5. Fluorescence property of HAp coating, HAp/tryptophan complex coating and HAp/phenylalanine complex coating at different excitation wavelength.

During the immersion testing, unreacted amino acid powder layer was removed and more area of the HAp/amino acid ligands were exposed. High pressure during CIP promoted molecular orientation of ligands onto the surface of HAp coating and subsequently influenced fluorescent property by increased intermolecular interactions. Such the molecular orientation consequently resulted in red-shift in fluorescent property using same molecules [43-49].

\subsection{Surface Roughness Measurement by AFM}

After accomplishing CIP process, surface roughness was observed by the atomic force microscope in dynamic mode. Result demonstrated that surface roughness increased after the CIP process due to the molecular interlocking of the contacting molecules. For the Ti-6Al-4V plate the surface roughness was within 5-10 $\mathrm{nm}$. After the electrochemical deposition method, the surface roughness of HAp coated specimen increased to 50-60 $\mathrm{nm}$ and after the CIP process for the surface roughness of HAp/tryptophan complex and 
HAp/phenylalanine complex increased to 70-75 $\mathrm{nm}$. The reason for the complex formation can be attributed to the intermolecular reaction and mechanical interlocking during the process of CIP that resulted from the compressive force applied [47-50].
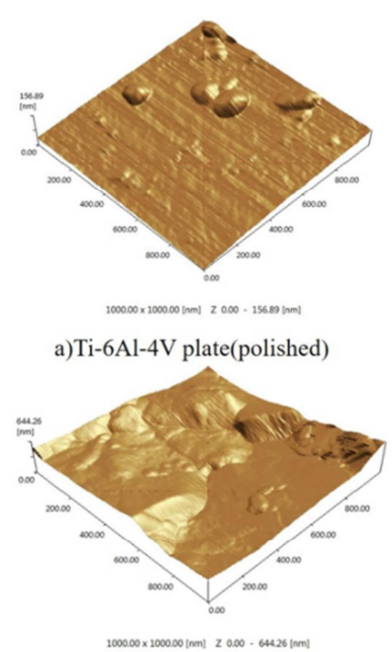

c)HAp/tryptophan complex

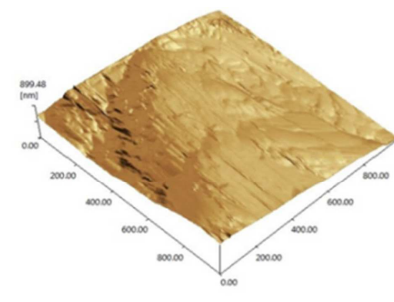

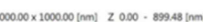

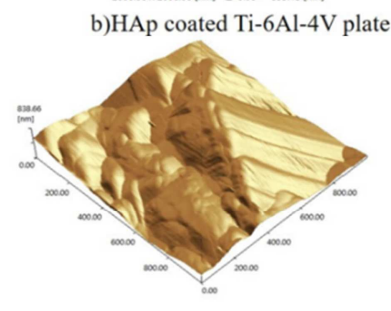

d)HAp/phenylalanine complex

Figure 6. Surface profile of a) polished Ti-6Al-4V plate, b) electrochemically deposited HAp coated plate, c) HAp/tryptophan complex coating and d) HAp/phenylalanine complex coating.

The Figure 6 shows the surface profile of a) polished Ti-6Al-4V plate, b) electrochemically deposited HAp coated plate, c) HAp/tryptophan complex coating and d) HAp/phenylalanine complex coating. The increased surface roughness indicated the formation of the newly formed complex.

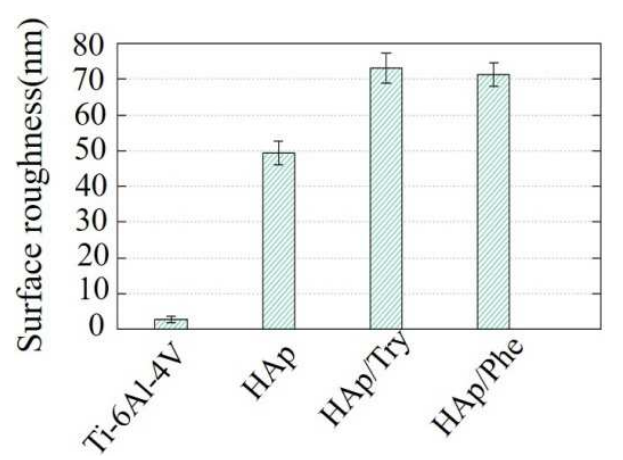

Figure 7. Surface roughness for four types of substrates.

Figure 7 showed the graphical presentation of the surface roughness evaluation after the complex was formed. The surface roughness was increased immensely in terms of HAp/amino acid complexes than the pure HAp coating and Ti-6Al-4V.

\subsection{Force Curve Measurement of Osteoblasts Cell by AFM}

AFM force measurement is the measurement of receptor-ligand interaction between a cell and an opposing substrate coating. First, the cantilever is lowered onto an osteoblasts cell and contact is made, allowing for the

receptor-ligand interaction to take place. Then, for pulling or breaking the receptor-ligand bond, the cantilever is retracted via the contraction of the piezoelectric translator. Finally, complete separation is achieved, later on the process can be repeated again. Throughout the approach and retraction events, the cantilever is bent and the tension between the cell and cantilever is determined from the deflection of the cantilever. AFM studies involving multiple bond interactions and these provide a great amount of valuable information about a particular receptor-ligand system.

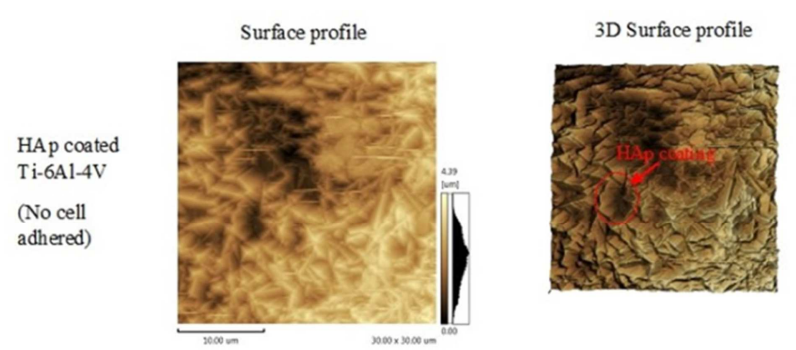

HAp coated
Ti-6Al-4V
(With
adhered cell)
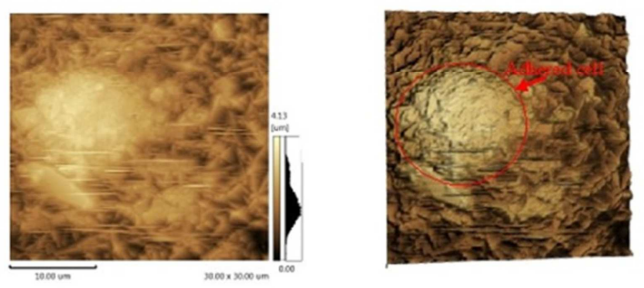

\section{HAp/tryptoph an complex coating \\ (With adhered cell)}
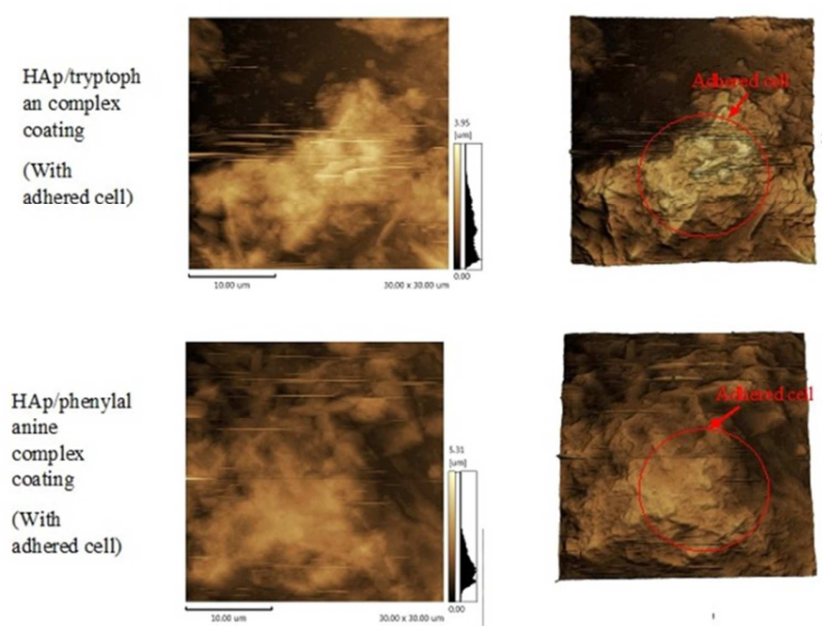

Figure 8. Surface profile of a) HAp coating with no adhered cell, b) HAp coated Ti-6Al-4V with adhered Osteoblasts cell, b) HAp/tryptophan complex coating with adhered Osteoblasts cell and d) HAp/phenylalanine complex coating with adhered Osteoblasts cell.

Force curve measurement was done after $24 \mathrm{hrs}$. cell culture. Figure 8 shows the surface profile of a) HAp coating with no adhered cell, b) HAp coated Ti-6Al-4V with adhered Osteoblasts cell, b) HAp/tryptophan complex coating with adhered Osteoblasts cell and d) HAp/phenylalanine complex coating with adhered Osteoblasts cell. Each case showed Surface profile with the 3D surface profile. From the force curve measurement Young's modulus value is calculated. Table 1 showed mechanical properties of different coating surfaces. 
Table 1. Mechanical Properties of different coating surfaces.

\begin{tabular}{lll}
\hline Type of Coated Surface & Surface Roughness, Ra (nm) & Young's Modulus (kPa) \\
\hline HAp coated Ti-6Al-4V & $56 \pm 2.9$ & $2.3 \pm 0.42$ \\
HAp/tryptophan complex coating & $74 \pm 3.5$ & $4.7 \pm 0.37$ \\
HAp/phenylalanine complex coating & $73.5 \pm 3.1$ & $5.2 \pm 0.49$ \\
\hline
\end{tabular}

The Young's modulus value for Osteoblasts cells were different but within the range of $0.3-20 \mathrm{kPa}$ for the applied force of $0.4 \mathrm{nN}$ [37]. Subsequently, slight variation in Young's modulus value can be due to the variation on the coated surface [38]. Newly fabricated HAp/amino acid complex coating didn't refer significant change in the mechanical properties and it can be concluded that the adhered cells were having similar mechanical properties as the cells adhered on HAp coating.

\subsection{Cell Morphology by SEM Observation}

Figure 9 shows surface profile of a) bare HAp coating, Osteoblasts cells adhered on b) HAp coated surface, c) HAp/tryptophan complex coating and d) HAp/phenylalanine complex coating. In case of bare HAp coated surface, the coating morphology refers to needle like structure. Adhered cells on HAp coated surface showed smaller size and more circular shape.
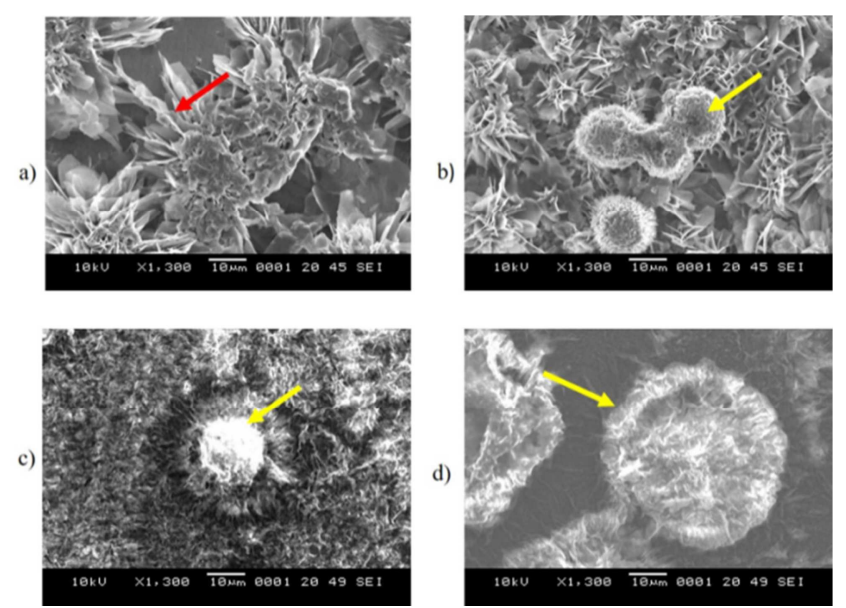

Figure 9. Surface profile of a) bare HAp coating, Osteoblasts cells adhered on b) HAp coated surface, c) HAp/tryptophan complex coating and d) HAp/phenylalanine complex coating ${ }^{*}$ Red arrow indicating needle like HAp coating and yellow arrow indicating cell body.

For cells adhered on complex coating, shape is larger for HAp/phenylalanine complex but for HAp/tryptophan complex the cell size and shape didn't change significantly in comparison with the HAp coated surface. The figure demonstrated that the cell has spread its filaments and the bonding between the cellular filament and the coated surface area was well oriented for HAp/phenylalanine complex coating. For Osteoblasts cells adhered on the HAp/tryptophan complex coating, the cell filaments were adhered but cell-cell interaction is less. This shows that both of these complexes have no significant difference for the cell adhesion behavior. Resulted observation clearly showed, even after AFM observation cells were present on the coated surface and the observed images resembles the shape of cells observed on AFM 3D surface profile with the SEM cellular images.

\subsection{EDS Analysis of Organic Elements}

The EDS mapping in Figure 10 shows clear evidence for the presence of organic essential elements for cellular body.

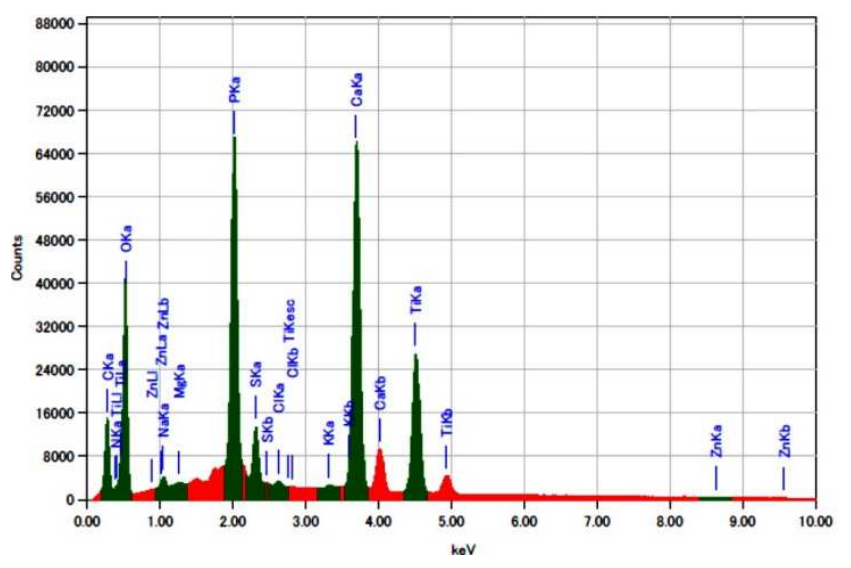

Figure 10. EDX analysis to confirm the presence of organic elements.

EDX analysis (Figure 11) showed presence of C, Ca, P, N elements. These elements are the significant compounds for the existence of any organic body.
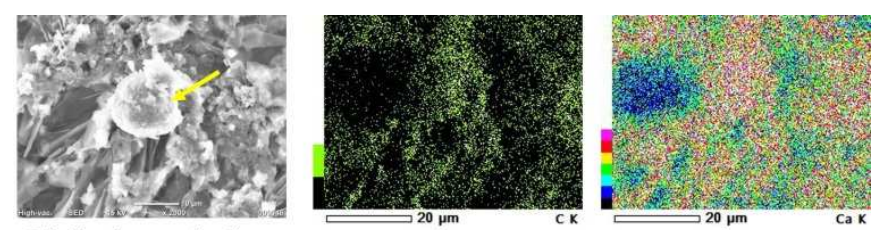

Cell adhered on coated surface
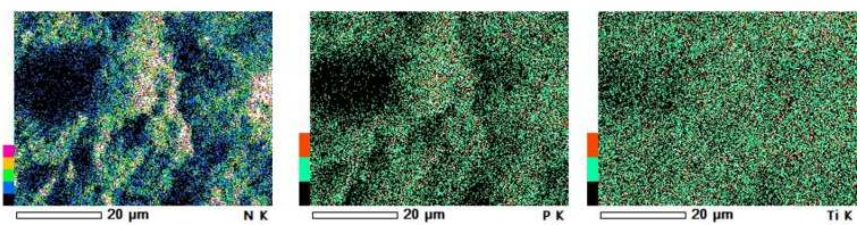

Figure 11. EDX analysis for the presence of cellular compounds. *Yellow arrow indicating cell body adhered on the coated surface.

Observed result demonstrated that these organic elements are those essential elements that are consequently, required for the presence of organic cellular body.

\section{Discussion}

Cold Isostatic Pressing method for fabricating fluorescent complexes between HAp and an amino acid was successfully developed. The HAp/phenylalanine complex after immersion showed red-shifted fluorescence. This 
exhibited the effect of Cold Isostatic Pressing pressure on promoting molecular orientation of amino acid ligand and that also change in the fluorescent property. The red-shift fluorescence is accredited to positioning of Phenylalanine molecules onto the surface of HAp coating [41, 48-52], and Cold Isostatic Pressing process can exclusively promote such an arrangement. Additionally, the HAp/amino acid complexes were nontoxic to mouse osteoblast like cells [44-47], these characteristics is desirable for multifunctional coating layers. This study visualized and characterized cell adhesion behavior with respect to mechanical properties of murine osteoblasts cell (MC3T3-E1) by AFM adhered on different biomaterial coating surface to determine the effect of cell adhesion on HAp/amino acid complex coating. It was found that the cells were well spread and for complex coating lengthened shape was obtained. In this experiment, we did not have a large number of cells because in our study conditions AFM depicted only a small area compared with the cell size (maximum, $30 \times 30 \times 5.5 \mu \mathrm{m}$ on $\mathrm{X}, \mathrm{Y}, \mathrm{Z}$ directions). Furthermore, cell adherence observation was limited to $5 \mathrm{~h}$, i.e., the cell lifetime for an AFM experiment. Even though several different areas were observed, the number of cells experiential was very small.

The measured average Young's modulus value for the osteoblasts cell for HAp coating was 2-3 kPa for HAp/tryptophan complex coating was $4-5 \mathrm{kPa}$ and or the HAp/phenylalanine complex coating was 4-6 $\mathrm{kPa}$. These values were within the range of $2-9 \mathrm{kPa}$ as the previous studies of Osteoblasts cells that were well adhered on different biomaterial surfaces [38-44]. Moreover, fluctuation in the value of Young's modulus also depends on various parameters i.e. surface roughness, relative humidity, measuring medium [49-51]. The surface observation shown in the AFM images (Figure 8) showed presence of cell during scanning. This is correlated with the result obtained from the SEM (Figure 9) observation also. So, here the result surely showed that the cellular body was detected during the AFM observation. Moreover, EDX analysis (Figure 11) confirms the presence of organic compounds that are the important features for cell body. The fabricated complex coating of HAp/amino acid ligands did not have any significant difference with cell adhesion process in comparison with the HAp coating deposited by the electrochemical deposition method.

The drawback of this research was the needle like structure of the HAp coating, further HAp coating process should be developed that will be suitable for the body cell as well as AFM analyzation. During AFM measurement fluctuation occurred for cell measurement process, so better adhesion process should be applied for later measurement. Force curve measurement result demonstrated typical fluctuations in contact region possibly due to viscoelasticity of cells. However, adhesive force can be influenced both the cell/cantilever interface and the cell/substrate interface. It is then necessary to consider the way of detecting detachment at different interfaces. Immobilization of cells is one of the possible solutions. Selection of cantilever, fixation method and force curve measurement method to selectively detect interfacial fracture between HAp coating with the cells should be further considered [31]. The present electrochemical technique can control the thickness of the HAp coating however, the coating strength is low in comparison with the other coating processes like plasma spraying process, suspension plasma spraying process etc. It is important to improve the strength of the coating surface but as well as control the thickness of the coating. Another limitation is that the measurement technique only gives approximate value. The measurement technique needs to be improved by considering other forces like lateral drag forces, van der walls forces etc. In this measurement process, the light irradiation was not considered. For the improvement of the effect of light irradiation and temperature effect should be considered. Fluorescent microscope unfortunately revealed that inhomogeneous complex layer was formed, which prevent us in quick measurement of cell adhesion behavior onto their surfaces. Further technology to fabricate homogeneous coating layer is then needing.

Adhesion force measurement is an important parameter to discuss the adhesion properties of cells. With this procedure, it is possible to determine the mechanical properties of different cells. This information provided by the force curve measurement can define the strength of the cells. More basic study for investigating adhesive strength of osteoblast cells quantitatively is necessary.

\section{Conclusion}

AFM probing is an effective tool to investigate cell elasticity and dynamics of cell mechanics. For example, characteristics of migrating cells, mechanical properties like Young's modulus evaluation is important to certify the diseased cell and normal cell.

In this research, electrochemical deposition process successfully produced HAp coating over the Ti-6Al-4V substrate and Cold Isostatic Pressing process successfully fabricated complexes of HAp with amino acid ligands as a fluorescent coating that is biocompatible and stable for use in human fluids in vivo. The HAp/amino acid complexes were retained in a liquid environment, and osteoblasts cells could grow on the surfaces just as the HAp coating. Moreover, there was no significant changes in the cellular mechanical properties regardless of complex coating variation. This newly developed complex coating could be one of the best candids for dental implant for application. For future perspective lateral force microscopy, phase imaging can be useful for the evaluation of spatial distribution of mechanical and structural characteristics of cell body.

\section{Acknowledgements}

The author would like to thank Echigoseika Co. Ltd for the use of a CIP machine and Ministry of Education, Culture, Sports, Science and Technology (MEXT), Japan for their 
kind support for doing the research. I am really thankful to Miyashita-Otsuka Laboratory of Nagaoka University of Technology to permit to use the SPM-9700, SEM and XRD machine with other experimental help.

\section{References}

[1] Ketaki Deshmukh, Sutapa Roy Ramanan, Meenal Kowshik. Novel one step transformation method for Escherichia coli and Staphylococcus aureus using arginine-glucose functionalized hydroxyapatite nanoparticles. Materials Science and Engineering: C, 96, 58-65, 2019.

[2] Mitsuo Niinomi. Mechanical properties of biomedical titanium alloys. Materials Science and Engineering: A, 243 (1-2): 231-236, 1998.

[3] Mitsuo Niinomi. Mechanical biocompatibilities of titanium alloys for biomedical applications. Journal of the Mechanical Behavior of Biomedical Materials, 1 (1): 30-42, 2008.

[4] T. W. Bauer and J. Schils. The pathology of total joint arthroplasty ii. Mechanisms of implant failure. Skeletal radiology, 28 (9): 483-497, 1999.

[5] A. Remes and D. F. Williams. Immune response in biocompatibility. Biomaterials, 13 (11): 731-743, 1992.

[6] B. 'R'ihov'a. Biocompatibility of biomaterials: Hemocompatibility, immunocompatibility and biocompatibility of solid polymeric materials and soluble targetable polymeric carriers. Advanced Drug Delivery References 52 .

[7] E. Fournier, C. Passirani, C. N. Montero-Menei, and J. P. Benoit. Biocompatibility of implantable synthetic polymeric drug carriers: Focus on brain biocompatibility. Biomaterials, 24 (19): 3311-3331, 2003.

[8] P. Thevenot, W. Hu, and L. Tang. Surface chemistry influences implant biocompatibility. Current Topics in Medicinal Chemistry, 8 (4): 270-280, 2008.

[9] F. Var'1ola, F. Vetrone, L. Richert, P. Jedrzejowski, J. -H. Yi, S. Zalzal, S. Clair, A. Sarkissian, D. F. Perepichka, J. D. Wuest, F. Rosei, and A. Nanci. Improving biocompatibility of implantable metals by nanoscale modification of surfaces: An overview of strategies, fabrication methods, and challenges. Small, 5 (9): 996-1006, 2009.

[10] J. D. Bryers, C. M. Giachelli, and B. D. Ratner. Engineering biomaterials to integrate and heal: The biocompatibility paradigm shifts. Biotechnology and Bioengineering, 109 (8): 1898-1911, 2012.

[11] S. Agarwal, J. Curtin, B. Duffy, and S. Jaiswal. Biodegradable magnesium alloys for orthopaedic applications: A review on corrosion, biocompatibility and surface modifications. Materials Science and Engineering C, 68: 948-963, 2016.

[12] L. Reeve and P. Baldrick. Biocompatibility assessments for medical devices-evolving regulatory considerations. Expert Review of Medical Devices, 14 (2): 161-167, 2017.

[13] Jr. Aduba, D. C. and H. Yang. Polysaccharide fabrication platforms and biocompatibility assessment as candidate wound dressing.

[14] D. A. Mbeh, R. Franac, a, Y. Merhi, X. F. Zhang, T. Veres, E.
Sacher, and L. Yahia. In vitro biocompatibility assessment of functionalized magnetite nanoparticles: Biological and cytotoxicological effects. Journal of Biomedical Materials Research-Part A, 100 A (6): 1637-1646, 2012.

[15] Robert B. Heimann. Thermal spraying of biomaterials. Surface and Coatings Technology, 201 (5): 2012-2019, 2006.

[16] J. H. Kim, S. H. Kim, H. K. Kim, T. Akaike, and S. C. Kim. Synthesis and characterization of hydroxyapatite crystals: A review study on the analytical methods. Journal of Biomedical Materials Research, 62 (4): 600-612, 2002.

[17] S. Overgaard, U. Bromose, M. Lind, C. B?nger, and K. S?balle. The influence of crystallinity of the hydroxyapatite coating on the fixation of implants. mechanical and histomorphometric results. Journal of Bone and Joint Surgery-Series B, 81 (4): 725-731, 1999.

[18] Carlos Nelson Elias, Felipe Assis Rocha, Ana Lucia Nascimento, and Paulo Guilherme Coelho. Influence of implant shape, surface morphology, surgical technique and bone quality on the primary stability of dental implants. Journal of the Mechanical Behavior of Biomedical Materials, 16: 169-180, 2012.

[19] Iman Hejazi, Javad Seyfi, Ehsan Hejazi, Gity Mir Mohamad Sadeghi, Seyed Hassan Jafari, and Hossein Ali Khonakdar. Investigating the role of surface micro/nano structure in cell adhesion behavior of superhydrophobic polypropylene/nanosilica surfaces. Colloids and Surfaces B: Biointerfaces, 127: 233-240, 2015.

[20] Stuart B. Goodman, Zhenyu Yao, Michael Keeney, and Fan Yang. The future of biologic coatings for orthopaedic implants Biomaterials,

[21] Jordan Raphel, Mark Holodniy, Stuart B. Goodman, and Sarah C. Heilshorn. Multifunctional coatings to simultaneously promote osseointegration and prevent infection of orthopaedic implants. Biomaterials, 84: 301-314, 2016.

[22] Hiroyuki Akiyama and Nobuhiro Hata. Introduction to Scanning Probe microscope. Ohmsha, Ltd., 2013.

[23] G. H. Enevoldsen, T. Glatzel, M. C. Christensen, J. V. Lauritsen, and F. Besenbacher. Atomic scale kelvin probe force microscopy studies of the surface potential variations on the $\mathrm{TiO}_{2}$ (110) surface. Phys. Rev. Lett., 100: 236104, Jun 2008 .

[24] Claudio Canale, Alessia Petrelli, Marco Salerno, Alberto Diaspro, and Silvia Dante. A new quantitative experimental approach to investigate single cell adhesion on multifunctional substrates. Biosensors and Bioelectronics, 48 (Supplement C): 172-179, 2013.

[25] E. Birkenhauer and S. Neethirajan. Characterization of electrical surface properties of mono-and co-cultures of pseudomonas aeruginosa and methicillin-resistant staphylococcus aureus using kelvin probe force microscopy. RSC Advances, 4 (80): 42432-42440, 2014.

[26] Tze-Wen Chung, Der-Zen Liu, Sin-YaWang, and Shoei-ShenWang. Enhancement of the growth of human endothelial cells by surface roughness at nanometer scale. Biomaterials, 24 (25): 4655-4661, 2003.

[27] X. Yao, J. Walter, S. Burke, S. Stewart, M. H. Jericho, D. Pink, R. Hunter, and T. J. Beveridge. Atomic force microscopy and theoretical considerations of surface properties. 
[28] N'uria Gavara. A beginner's guide to atomic force microscopy probing for cell mechanics. Microscopy Research and Technique, 80 (1): 75-84, 2017.

[29] Junjian Chen, Yuchen Zhu, Yancheng Song, Lin Wang, Jiezhao Zhan, Jingcai He, Jian Zheng, Chunting Zhong, Xuetao Shi, Sa Liu, Li Ren, and YingjunWang. Preparation of an antimicrobial surface by direct assembly of antimicrobial peptide with its surface binding activity. J. Mater. Chem. B, 5: 2407-2415, 2017.

[30] F. Zuttion, C. Ligeour, O. Vidal, M. Walte, F. Morvan, S. Vidal, J.-J. Vasseur, Y. Chevolot, M. Phaner-Goutorbe, and H. Schillers. The anti-adhesive effect of glycoclusters on: Pseudomonas aeruginosa bacteria adhesion to epithelial cells studied by afm single cell force spectroscopy. Nanoscale, 10 (26): 12771-12778, 2018 .

[31] Wen Zhang, Andrew G. Stack, and Yongsheng Chen. Interaction force measurement between e. coli cells and nanoparticles immobilized surfaces by using afm. Colloids and Surfaces B: Biointerfaces, 82 (2): 316-324, 2011.

[32] Q. Huang, H. Wu, P. Cai, J. B. Fein, and W. Chen. Atomic force microscopy measurements of bacterial adhesion and biofilm formation onto clay-sized particles. Scientific Reports, $5,2015$.

[33] M. Tanaka, T. Hayashi, and S. Morita. The roles of water molecules at the biointerface of medical polymers. Polymer Journal, 45 (7): 701-710, 2013.

[34] G. T. Charras, P. P. Lehenkari, and M. A. Horton. Atomic force microscopy can be used to mechanically stimulate osteoblasts and evaluate cellular strain distributions. Ultramicroscopy, 86 (1-2): 85 .

[35] D. Docheva, D. Padula, C. Popov, W. Mutschler, H. Clausen-Schaumann, and M. Schieker. Researching into the cellular shape, volume and elasticity of mesenchymal stem cells, osteoblasts and osteosarcoma cells by atomic force microscopy: Stem cells. Journal of Cellular and Molecular Medicine, 12 (2): 537-552, 2008.

[36] J. Domke, S. Dann“ohl, W. J. Parak, O. M"uller, W. K. Aicher, and M. Radmacher. Substrate dependent differences in morphology and elasticity of living osteoblasts investigated by atomic force microscopy. Colloids and Surfaces B: Biointerfaces, 19 (4): 367-379, 2000.

[37] A. Simon, T. Cohen-Bouhacina, M. C. Port'e, J. P. Aim'e, J. Am'ed'ee, R. Bareille, and C. Baquey. Characterization of dynamic cellular adhesion of osteoblasts using atomic force microscopy. Cytometry Part A, 54 (1): 36-47, 2003.

[38] E. Takai, K. D. Costa, A. Shaheen, C. T. Hung, and X. E. Guo. Osteoblast elastic modulus measured by atomic force microscopy is substrate dependent. Annals of Biomedical Engineering, 33 (7): 963-971, 2005.

[39] Federico Caneva Soumetz, Jose F. Saenz, Laura Pastorino, Carmelina Ruggiero, Daniele Nosi, and Roberto Raiteri. Investigation of integrin expression on the surface of osteoblast-like cells by atomic force microscopy. Ultramicroscopy, 110 (4): 330-338, 2010.

[40] Y. Yamato, M. Matsukawa, H. Mizukawa, T. Yanagitani, K. Yamazaki and A. Nagano, Distribution of hydroxyapatite crystallite orientation and ultrasonic wave velocity in ring-shaped cortical bone of bovine femur. IEEE Transactions on Ultrasonics, Ferroelectrics, and Frequency Control, 55 (6), 1298-1303, 2008.

[41] Adnan Haider, Sajjad Haider, Sung Soo Han, Inn-Kyu Kang. Recent advances in the synthesis, functionalization and biomedical applications of hydroxyapatite: a review. RSC Advances, 7 (13), 7442-7458, 2017.

[42] Cosmin M. Cotrut, Alina Vladescu, Mihaela Dinu, and Diana M. Vranceanu. Influence of deposition temperature on the properties of hydroxyapatite obtained by electrochemical assisted deposition. Ceramics International, 44 (1): 669-677, 2018.

[43] Somtirtha Banerjee, Biswajoy Bagchi, Suman Bhandary, Arpan Kool, Nur Amin Hoque, Prosenjit Biswas, Kunal Pal, Pradip Thakur, Kaustuv Das, Parimal Karmakar, Sukhen Das. Antimicrobial and biocompatible fluorescent hydroxyapatite-chitosan nanocomposite films for biomedical applications. Colloids and Surfaces B: Biointerfaces, 171, 300-307, 2018.

[44] Ewa P. Wojcikiewicz, Xiaohui Zhang, and Vincent T. Moy. Force and compliance measurements on living cells using atomic force microscopy (afm). Biological Procedures Online, 6 (1): 1-9, Jan 2004.

[45] A. Hashimoto, Y. Yamaguchi, L.-D. Chiu, C. Morimoto, K. Fujita, M. Takedachi, S Kawata, S. Murakami, and E. Tamiya. Time-lapse raman imaging of osteoblast differentiation. Scientific Reports, 5, 2015.

[46] L. Sun, C. C. Berndt, K. A. Gross, A. Kucuk, Material fundamentals and clinical performance of plasma-sprayed hydroxyapatite coatings: a review, J. Biomed. Mater. Res. 58 (5) (2001) 570-592.

[47] R. B. Heimann, Thermal spraying of biomaterials, Surf. Coat. Technol. 201 (5) (2006) 2012-2019.

[48] K. Ozeki, Y. Fukui, H. Aoki, Hydroxyapatite coated dental implants by sputtering technique, Biocybernetics Biomed. Eng. 26 (1) (2006) 95-101.

[49] M. Wei, A. Ruys, B. Milthorpe, C. Sorrell, J. Evans, Electrophoretic deposition of hydroxyapatite coatings on metal substrates: a nanoparticulate dual-coating approach, J. Sol-Gel. Sci. Technol. 21 (1) (2001) 39-48.

[50] Atyana G. Kuznetsova, Maria N. Starodubtseva, Nicolai I. Yegorenkov, Sergey A. Chizhik, Renat I. Zhdanov, Atomic force microscopy probing of cell elasticity, Micron, Volume 38 , Issue 8, 2007, Pages 824-83351.

[51] Alam, Fahad \& Balani, Kantesh. (2016). Adhesion Force of Staphylococcus aureus on Various Biomaterial Surfaces. Journal of the Mechanical Behavior of Biomedical Materials.

[52] Shuli Zeng, Ronghui Zhou, Xiaoke Zheng, Lan Wu, Xiandeng Hou. Mono-dispersed $\mathrm{Ba}$ 2+-doped Nano-hydroxyapatite conjugated with near-infrared $\mathrm{Cu}$-doped $\mathrm{CdS}$ quantum dots for $\mathrm{CT} /$ fluorescence bimodal targeting cell imaging. Microchemical Journal, 134, 41-48, 2017. 